\title{
Language in Amnestic Mild Cognitive Impairment and Dementia of Alzheimer's Type: Quantitatively or Qualitatively Different?
}

\author{
Regina Jokel ${ }^{\mathrm{a}-\mathrm{c}}$ Bruna Seixas Limac ${ }^{c}$ Alita Fernandez ${ }^{\mathrm{a}}$ Kelly J. Murphy ${ }^{\mathrm{c}-\mathrm{e}}$ \\ a Rotman Research Institute, Toronto, ON, Canada; b Speech-Language Pathology, Baycrest \\ Health Sciences, Toronto, ON, Canada; ' University of Toronto, Toronto, ON, Canada; \\ dNeuropsychology and Cognitive Health, Baycrest Health Sciences, Toronto, ON, Canada; \\ e Psychology Department, York University, Toronto, ON, Canada
}

\section{Keywords}

Mild cognitive impairment - Language · Alzheimer's disease

\begin{abstract}
Background/Aims: The purpose of this study was to explore language differences between individuals diagnosed with amnestic mild cognitive impairment multiple domain (aMClm) and those with probable Alzheimer's disease, with a goal of (i) characterizing the language profile of aMCIm and (ii) determining whether the profiles of dementia of Alzheimer's type (DAT) and aMClm individuals are on a continuum of one diagnostic entity or represent two distinct cognitive disorders. Methods: Language data from 28 patients with consensus diagnosis of aMClm and DAT derived from a retrospective chart review were compared to that of healthy controls. Results: A non-parametric statistic established that there was no significant difference between groups in age, years of education or duration of symptoms and that expressive language was found to be relatively intact in both patient groups. In contrast, both groups exhibited significant impairments on receptive language tests and on linguistically complex tasks that rely on episodic memory and executive functions. Individuals with aMCIm and DAT present with configurations of language features that are largely in parallel to each other and reflect predominantly quantitative differences. Conclusion: Language tests provide an important contribution to the diagnostic process in their capacity to identify language impairments at an early stage. Understanding the nature of language decline is critically important to the intervention process as this information would inform cognitive intervention approaches aimed at promoting quality of life in people living with $\mathrm{MCl}$ and dementia.
\end{abstract}


Jokel et al.: Language in $\mathrm{MCl}$ and DAT

\section{Introduction}

Episodic memory decline is an early and predominant symptom of probable Alzheimer's dementia [1-3]. As a consequence, given the prevalence of dementia, different types of memory have been extensively studied in individuals suspected of having Alzheimer's disease (AD). Less well studied are changes in language function (e.g., connected speech, naming ability, reading and writing skills) which may further inform both diagnostic and intervention approaches to identifying and treating people at risk of or suspected of having dementia of Alzheimer's type (DAT).

Researchers have effectively established clinical criteria to identify the earliest signs of possible DAT through adding to the general classification a cognitive disorder known as mild cognitive impairment (MCI) $[4,5]$. MCI differs from normal ageing due to the presence of greater than age-expected decline on cognitive tests as indicated by the test's normative data. It also differs from dementia because the cognitive decline is not significant enough to result in a marked loss of functional independence. The loss of functional independence (e.g., cooking, banking, driving) is equated in the clinical domain with transition from MCI towards dementia. The term "mild cognitive impairment" was originally used by Flicker et al. [6] to refer to a subset group of older adults who showed detectible cognitive decline over time. The study of Flicker et al. [6] and many subsequent studies provided evidence that alterations in cognitive functions (as well as in neurological integrity of the brain) can be observed years before the diagnosis of dementia. Petersen et al. [7] were the first to establish the classification criteria for the diagnosis of MCI, the refinement of which has evolved over time. These criteria refined after key symposia [4, 8] include the following: report of a cognitive complaint (self or informant); objective cognitive impairment (on testing); generally intact functional abilities (no significant functional impairment); and no presence of dementia (for review of clinical criteria, see [4, 5]). Albert et al. [5] outline in detail the criteria for MCI and AD, relating the MCI to prodromal AD. Those clinical and cognitive criteria underpin the differences between Alzheimer's pathology and $\mathrm{AD}$ as a syndrome. While the latter can be established with cognitive testing, the former requires further examination of the pathophysiological processes. In MCI that means excluding all vascular, traumatic and medical reasons, and there should be evidence of longitudinal decline. AD genetic history needs to be documented where relevant as this may assist in subsequent predictions regarding potential conversion to AD. In $\mathrm{AD}$, there is a list of biomarkers that need to be considered [5] in the process of differentiation between $\mathrm{AD}$ pathology and $\mathrm{AD}$ syndrome.

Although memory decline is the most common feature associated with $\mathrm{MCI}$, hence the term "amnestic" MCI, studies have shown that there are several other subtypes of MCI representative of different neurodegenerative pathologies and cognitive profiles. These subtypes are categorized based on memory status (amnestic versus non-amnestic) and on the number of cognitive domains involved (single domain versus multiple domain) (for reviews, see [4, 9].

In this study we are particularly interested in language profiles associated with amnestic mild cognitive impairment multiple domain (aMCIm) as they are clinically most resembling Alzheimer's dementia. Our goal was to linguistically compare the aMCIm population with that of DAT. We included only those participants who presented, to the best of our knowledge, with MCI due to $\mathrm{AD}$ pathology. In the absence of other possible causes of cognitive impairment (e.g., head injury, substance abuse, exposure to heavy metals, etc.), AD is often a suspected aetiology of MCI and there is a high conversion rate from amnestic MCI to AD [10-12].

In view of the focus of our paper, a special case of MCI should be mentioned here. It concerns a disorder which, from the neuropsychological standpoint, can be viewed as a nonamnestic MCI, namely the logopenic variant primary progressive aphasia (lvPPA). The 
emerging literature on lvPPA introduced it as a possible precursor to atypical AD or its linguistic variant [13-15]: lvPPA presents with naming impairment, phonemic errors in spontaneous speech and naming, and decreased ability on orally repeated sentences, while other aspects of language (semantics, syntax) remain intact [16]. Naming impairment and decreased sentence repetition, along with their purported mechanisms, are shared by dementia of the AD type [17]. Impaired access to phonology (word form) is connected to naming impairments while decreased working memory is thought to underlie the repetition impairment. We excluded lvPPA from our study despite its language features as we focused on the variant aMCIm.

The literature on lvPPA and language decline in dementia provides information on how language impairments, and connected speech in particular [18], interfere with cognitive function and thus can serve as a marker in dementia diagnosis. However, less is understood about the more general decline of language and its possible diagnostic value in MCI. Cognitively demanding tasks, such as reading, writing, cooking, working with a computer, talking on the phone or playing games, are just some examples of the daily activities that can be impaired due to language deficits leading to distress and depression $[19,20]$. The ability to carry out these types of daily living activities have been said to make a distinction between normal cognitive ageing, MCI and DAT [21]. It is not clear, however, which types of daily functions are more or less subtly affected in $\mathrm{MCI}$, as they can range from cognitive to motor to social skills. We would posit that the more complex the activity, the more vulnerable it will become to cognitive changes, and that language impairment would further enhance cognitive decline. Negotiating a mortgage rate may be more challenging than ordering from a menu at the restaurant, which, in turn, may be more demanding than responding to a greeting from a neighbour. While the latter can be accomplished in a semi-automatic manner, the mortgage negotiation will inadvertently be impacted by language impairment.

There are many subtle changes in cognitive function in MCI that have been documented in a number of studies. While word finding difficulties in conversation, confrontation naming and description tasks are present often and early in $\mathrm{AD}[22,23]$, lexical production in MCI has been found impaired only on tasks that require effortful semantic memory processing [24]. Similar to the AD individuals, MCI patients show a decline in semantic fluency when compared to phonemic fluency $[25,26$, respectively]. Those subtle changes, in addition to naming impairments, have been found useful in predicting the conversion from MCI to DAT [27-32]. Testing non-verbal semantics might be informative, to detect whether those subtle semantic deficits span both verbal and non-verbal domains. Verbal memory for newly learned words has also been shown to be impaired in MCI [33, 34].

Semantic knowledge outside the word and sentence is another cognitive impairment in MCI. MCI patients have presented with difficulty using pragmatic skills in tests of proverb meaning interpretation [35-37] and irony comprehension [38]. Although personal semantic knowledge appears to remain intact in MCI, autobiographical recollection of the episodic details associated with a happening experienced during a specific time and place are reduced when compared to same-aged controls [39]. Preserved language skills are critical for communicating autobiographical information, and a recent study investigating language and autobiographical memory in individuals with DAT and semantic dementia found that both groups produced an increased level of off-target present tense verbs where past tense verbs should be expected, begging an answer to the question of whether these deficits are a product or contributing factor in memory impairment [40]. Future research should examine these features in autobiographical recollection in MCI.

Worth noting is that relaying personal information is often associated with abilities necessary for discourse production (such as coherence and cohesion) and may possibly serve as an early marker of cognitive decline. The status of those abilities reflects potential problems 
in communication, organization skills and planning. Dijkstra etal. [41] compared the discourse features of typically ageing adults and adults with dementia, and found that the dementia patients' discourse presented more topic shifts and empty phrases than the healthy ageing participants, whose discourse was more coherent and cohesive. Harris et al. [42] have found in a discourse production task that MCI patients produced the least amount of thematic information (i.e., information about a certain theme) in a comparison between groups which included younger adults, typically ageing adults and people with neurological damage. Investigations of discourse length, complexity and quality have established that healthy older adults used more words than those with MCI. Individuals with MCI produced fewer core elements of the narrative elicited and showed word-retrieving deficits which were not always reflected in standard naming tasks, leading to the conclusion that the discourse production task was more challenging than the naming task [43]. In a study comparing discourse production between healthy controls, MCI patients and DAT patients, Drummond et al. [44] found that the MCI group's performance would at times resemble controls' (similar type of discourse, use of macropropositions and cohesion) and at times resemble the DAT group's performance (similar amount of irrelevant propositions).

In summary, linguistic behaviour has increasingly become a matter of interest in the search for cognitive markers of dementing states [45; for a review, see 46]. Language deficits in DAT have been widely documented; impairments have been found in verbal fluency [22, $23,25]$, naming $[47,48]$ and discourse [49], whereas syntactic and phonological abilities seem to remain preserved [22, 49 but see 50 and 51]. These deficits can be observed often and early in the course of DAT, which highlights the importance of investigating linguistic changes in MCI as a potential means to aid in diagnosis and, potentially, subcategorization. Characterization of language profiles may also assist in the prediction of conversion to dementia and, importantly, which type of dementia. Understanding the nature of how language processes are impacted by a suspected neurodegenerative disease of Alzheimer's type also provides critical information for cognitive intervention research aimed at promoting and sustaining aspects of functional independence in individuals living with $\mathrm{MCI}$ and DAT. For instance, identifying deficits in a person's orthographic processing will steer away any strategies relying on written messages.

Motivated by the continuous efforts to refine the diagnostic criteria and implement early diagnosis and intervention in dementia, we compare linguistic profiles of participants in three groups that included healthy individuals, those with the diagnosis of probable AD and aMCIm, respectively. The null hypothesis is that there are no statistically significant differences between the three groups. Given the documented rate of conversion between aMCI and dementia $[10-12,52]$ we hypothesized that we may find quantitative rather than qualitative differences between aMCIm and $\mathrm{AD}$ groups. We made no claims regarding other types of MCI.

\section{Materials and Methods}

\section{Participants}

This is a retrospective chart review of 28 individuals meeting current clinical diagnostic criteria for amnestic MCI with multiple areas of impairment (aMCIm, $n=14$ ) [4] and DAT $(n=14)$ [53]. The charts belong to patients from a memory clinic in Toronto. Diagnosis was established by multidisciplinary consensus between neuropsychologist, behavioural neurologist, neuropsychiatrist and speech-language pathologist, based on extensive clinical investigations, cognitive assessment, informant interview and structural neuroimaging. Importantly, only charts of individuals with the amnestic presentation of $\mathrm{MCI}$ in multiple cognitive domains were selected for the study to avoid those individuals with MCI single-domain who 
may have had language as the first and most impaired domain and thus would also fulfil criteria for primary progressive aphasia (PPA). Inevitably, all individuals with PPA would present with significant and predominant language impairment, which is defined by its own set of diagnostic criteria [16]. The functional status of included patients was also documented through activities of daily living questionnaire and confirmed with an informant. This information was extracted from each chart. All patients whose data were included in the study were either native English speakers or educated and/or fluent in English. Exclusion criteria consisted of history of untreated mental illness, prior head injury, cerebrovascular disease, alcoholism and/or drug addiction. We also recruited 12 healthy control participants with comparable mean age and education who were tested on the same set of language tests as those available from the aMCIm and DAT participants' charts. The demographic and language data for the three groups of participants are presented in Tables 1-3.

\section{Materials and Procedures}

Results of language tests administered to aMCIm and DAT participants between 2012 and 2017 were extracted from their medical files. Participants were diagnosed with MCI according to the criteria of Albert et al. [5], which include: (1) cognitive concern reflecting a change in cognition by patient and/or other informant; (2) objective evidence from neuropsychological tests; (3) full independence in all activities of daily living; and (4) no dementia. The aetiology of $\mathrm{MCI}$ was examined to establish consistency with AD pathophysiology and to rule out vascular, traumatic and medical causes. We took note of insidious onset and gradual progression, and genetic predispositions for $\mathrm{AD}$, if any. Participants with $\mathrm{AD}$ were diagnosed according to the criteria of McKhann et al. [53]. Consequently, their cognitive decline interfered with the ability to function at work or at usual activities, represented a decline from previous levels of functioning and performing, could not be explained by delirium or major psychiatric disorder, and was diagnosed through (a) history-taking from the patient and a knowledgeable informant and (b) an objective cognitive assessment. Additionally, the decline involved a minimum of two cognitive domains, plus insidious onset and no evidence of: (a) cerebrovascular disease, (b) core features of dementia with Lewy bodies, (c) behavioural variant frontotemporal dementia, (d) semantic variant PPA or non-fluent/agrammatic variant PPA, (e) evidence for another neurological disease or medication that could have an effect on cognition.

The test battery comprised tests that evaluated aspects of: (i) expressive language (oral and written), such as naming, sentence repetition, oral reading of paragraphs and words, and spelling of regular and irregular words; (ii) receptive language, such as word recognition and comprehension of grammar; (iii) verbal and non-verbal measures of semantics knowledge, and (iv) selected cognitive tasks that had a very strong language component, such as the MiniMental State Examination (MMSE) [54], verbal fluency, story retell and statements regarding orientation to time and place. In addition, information pertaining to motor speech (such as presence of apraxia or dysarthria) and the use of correct syntax in spontaneous speech was extracted from speech-language pathology reports. Tests included in the analysis, in part or in their entirety, consisted of the Boston Naming Test [55], parts of the Boston Diagnostic Aphasia Exam (sentence repetition, Cookie Theft - oral picture description) [56], Arizona Battery for Communication Disorders of Dementia (story retell) [57] subtests of Psycholinguistic Assessments of Language Processing in Aphasia (word reading and spelling) [58], Test for the Reception of Grammar (TROG) [59], Peabody Picture Vocabulary Test [60], Pyramids and Palm Trees Test [61], as well as phonemic and semantic fluency measures [62]. All MCI and AD participants whose charts were reviewed were tested by a speech-language pathologist from the memory clinic. All tests were completed during a single session and typically administered in the same order. All control participants were tested in 2017 by a research assistant. No experimental tasks were administered in this study. 
Dementia

and Geriatric

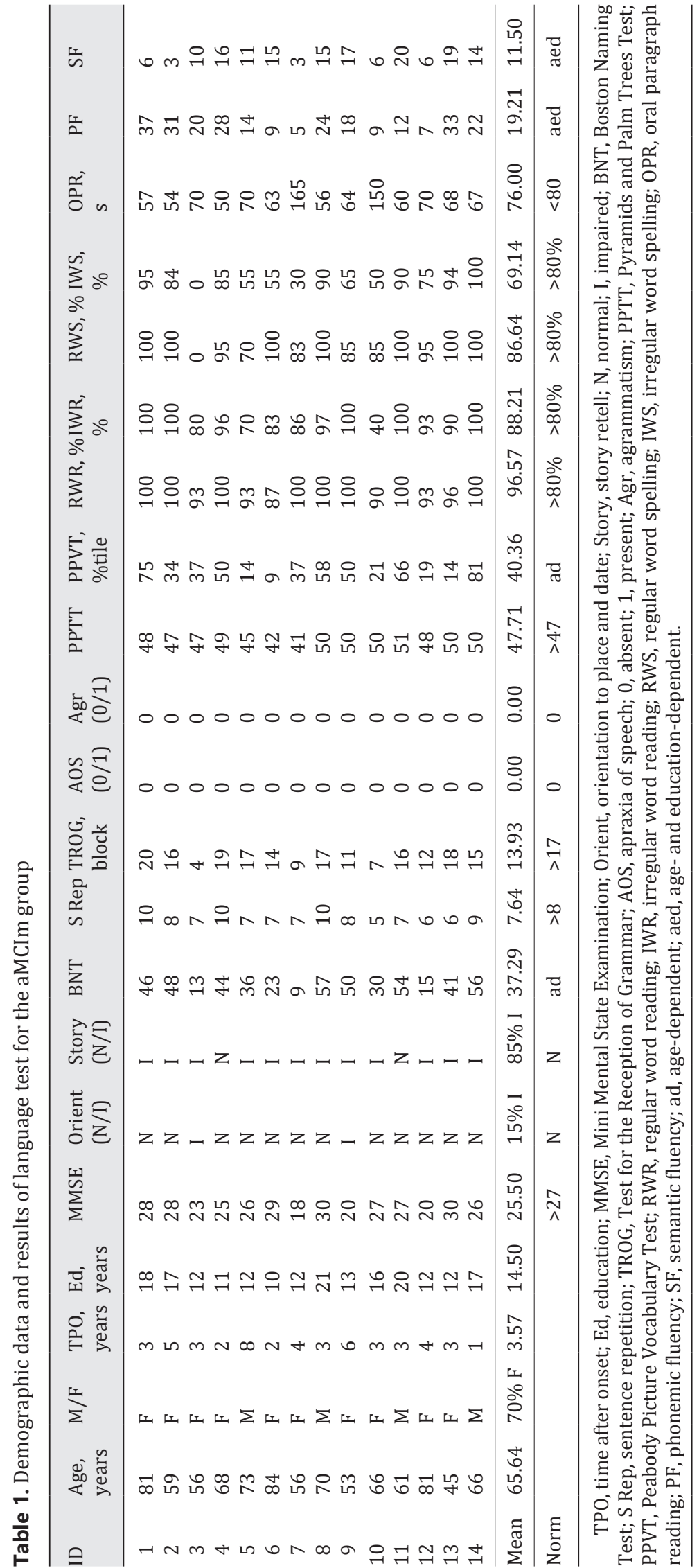




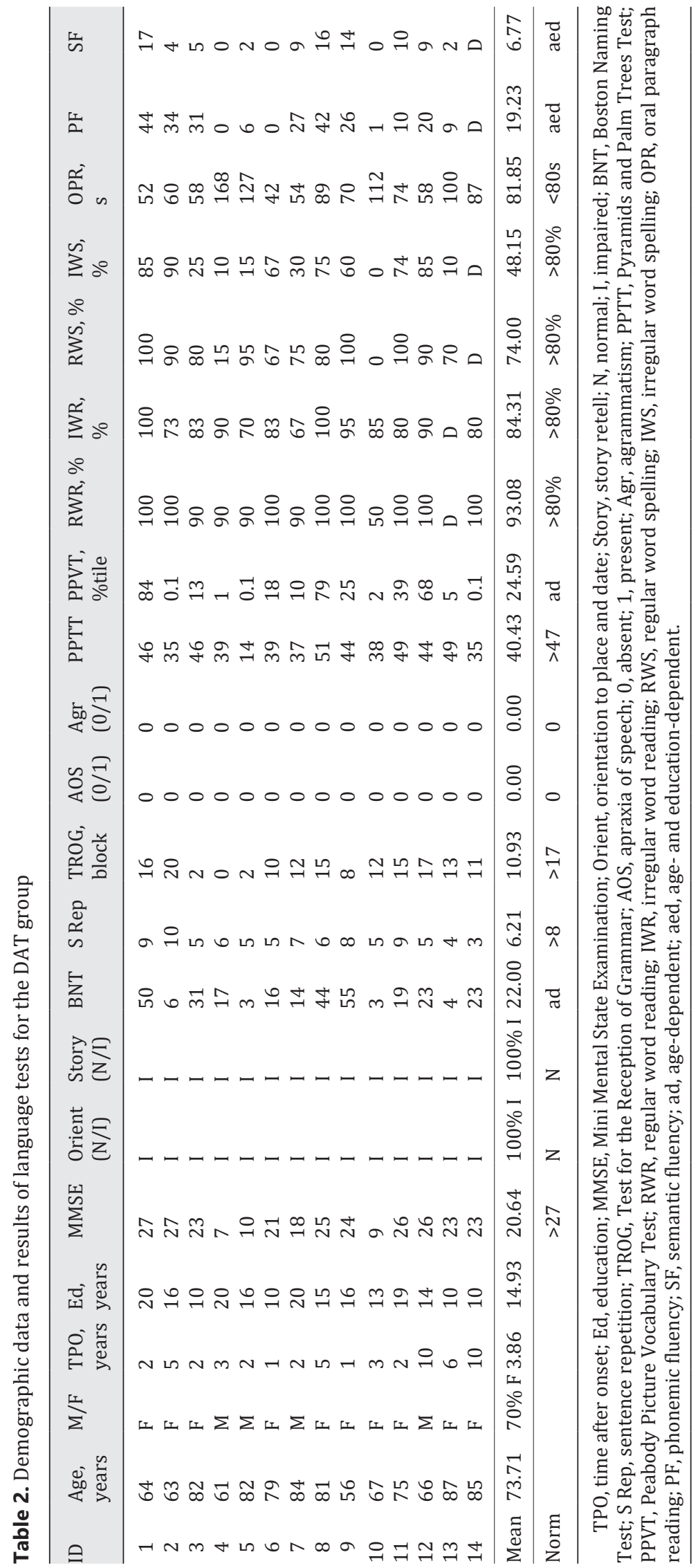


Dementia

and Geriatric
Dement Geriatr Cogn Disord Extra 2019;9:136-151

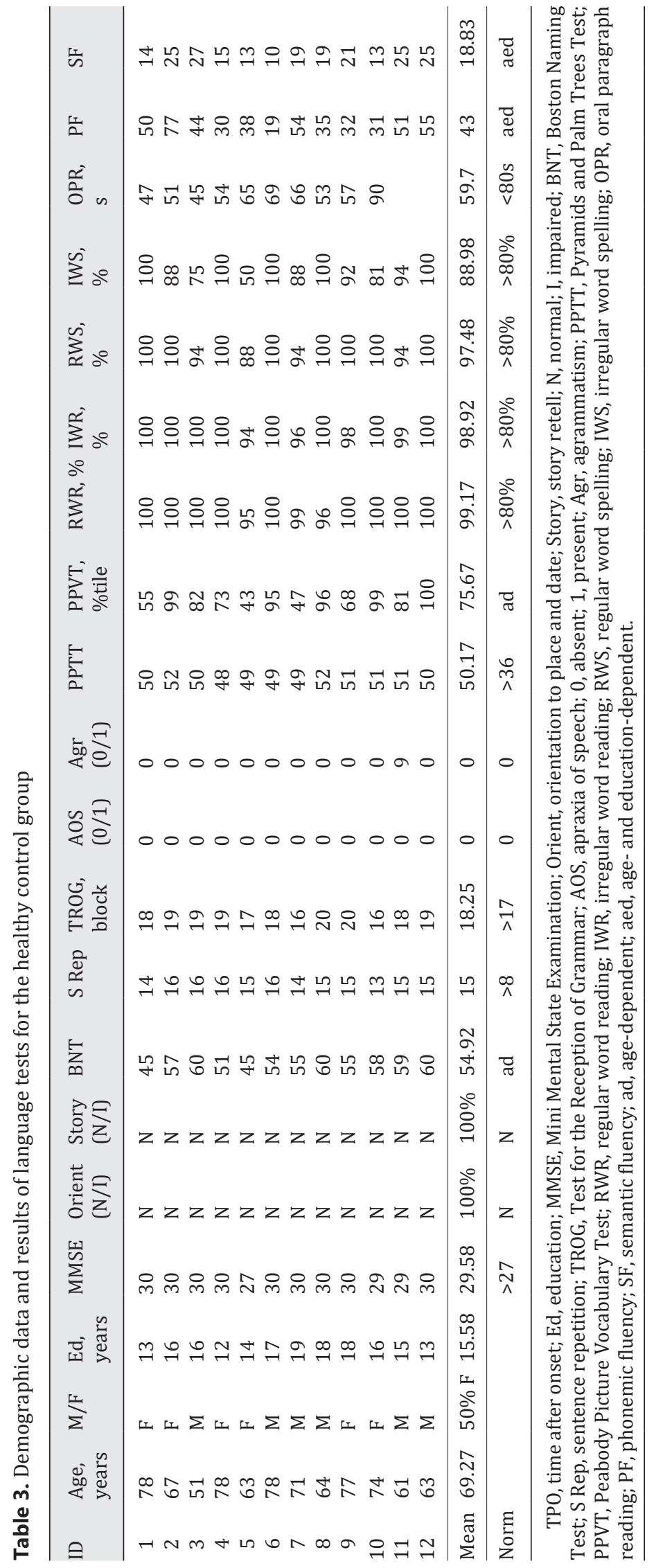


A non-parametric statistic, Kruskal-Wallis, was conducted to compare the results of language tests in the three groups. Bonferroni correction was applied to all multiple pair-wise comparisons. Published norms and control data for each administered test were utilized to determine whether each participant's averages were within the normal range. All data utilized in the review were de-identified, and approval was granted by the Baycrest Health Sciences Research Ethics Board.

\section{Results}

Demographic data and results of language tests for the aMCIm and DAT groups are presented in Tables 1 and 2, respectively. A Kruskal-Wallis statistic was conducted on all demographic and language data. All three groups were matched for age $(\mathrm{H}(2)=3.969, p=$ $0.137)$ and education $(\mathrm{H}(2)=0.848, p=0.654)$. The two patient groups were also matched for disorder duration $(p=0.589)$. The average time after onset in years was 3.9 (range 1-10) for the DAT group and 3.6 (range 1-8) for the aMCI group.

Analyses on language data revealed significant differences on some tasks between some groups. The Mann-Whitney U test was carried out on all significant findings, with the Bonferroni-adjusted $\alpha$-value of $\mathrm{p}<0.017$.

\section{Controls versus aMCIm}

Participants in the aMCIm group performed significantly worse than control participants on $\operatorname{MMSE}(\mathrm{U}=1.926, p<0.001)$ and a number of language measures. They included naming $(\mathrm{U}=1.430$, $p<0.001)$, sentence repetition $(\mathrm{U}=5.594, p<0.001)$, comprehension of grammar $(\mathrm{U}=1.674, p<$ $0.01)$, lexical semantic knowledge $(U=1.403, p<0.003)$, phonemic fluency $(U=1.807, p<0.001)$, semantic fluency $(\mathrm{U}=1.257, p<0.014)$ and verbal story recall $(\mathrm{U}=3.350, p<0.001)$. No differences emerged in orientation $(p=0.48)$, word reading $(p=0.26)$ or spelling $(p=0.41)$. The largest discrepancies occurred on naming, story retell, sentence repetition and phonemic fluency tests.

\section{Controls versus DAT}

Participants in the DAT group performed significantly lower than control participants on all tests administered, with the exception of regular word reading $(p=0.469)$. They were not well oriented to time and/or location ( $\mathrm{U}=\infty, p<0.001)$. Their MMSE showed impaired cognitive function $(\mathrm{U}=1.805, p<0.001)$ and identified impairments on all language tests. The DAT participants were impaired on naming $(\mathrm{U}=2.573, p<0.001)$, sentence repetition $(\mathrm{U}=$ $5.429, p<0.001)$, comprehension of grammar $(\mathrm{U}=1.674, p<0.001)$, irregular word reading $(\mathrm{U}=0.902, p<0.001)$, spelling of regular $(\mathrm{U}=1.038, p<0.005)$ and irregular words $(\mathrm{U}=1.572$, $p<0.001)$, non-verbal semantic knowledge $(\mathrm{U}=1.467, p<0.001)$, word knowledge $(\mathrm{U}=$ $2.145, p<0.001)$, phonemic fluency $(\mathrm{U}=1.515, p<0.001)$, semantic fluency $(\mathrm{U}=2.015, p<$ $0.001)$ and story retell $(\mathrm{U}=\infty, p<0.001)$.

\section{aMCIm versus DAT}

As evidenced in the above sections, both aMCIm and DAT groups presented with various impairments that set them apart from the control group. When it comes to the differences between the two patient groups, non-verbal semantic knowledge $(U=1.050, p<0.005)$ and orientation $(\mathrm{U}=3.350, p<0.001)$ appeared significantly worse in the DAT group at the $p<$ 0.0017 significance level while MMSE $(U=0.915, p=0.027)$, naming $(U=1.708, p=0.019)$, sentence repetition $(\mathrm{U}=0.771, p=0.021)$ and semantic fluency $(\mathrm{U}=0.786, p=0.041)$ were at the $p<0.05$ and did not reach the Bonferroni-adjusted significance level. A comprehensive summary of findings is presented in Table 4. 
Dementia

Cognitive Disorders

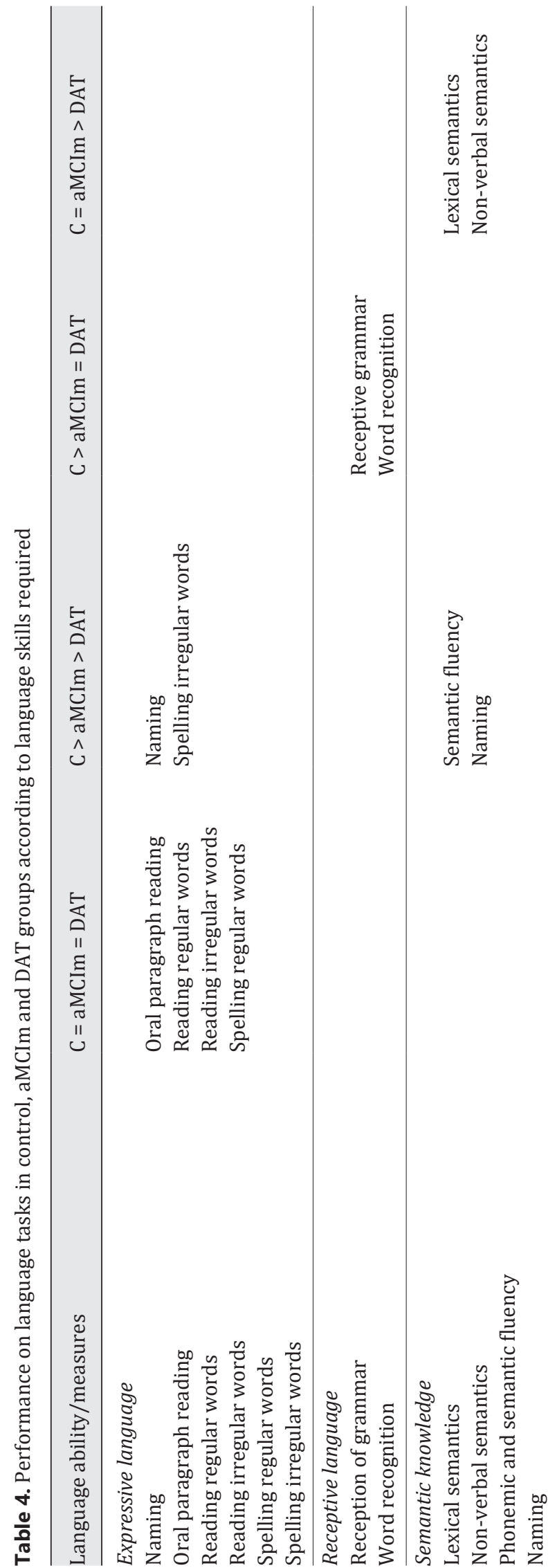

Dement Geriatr Cogn Disord Extra 2019;9:136-151

DOI: 10.1159/000496824

(c) 2019 The Author(s).P
www.karger.com/dee

Jokel et al.: Language in $\mathrm{MCl}$ and DAT

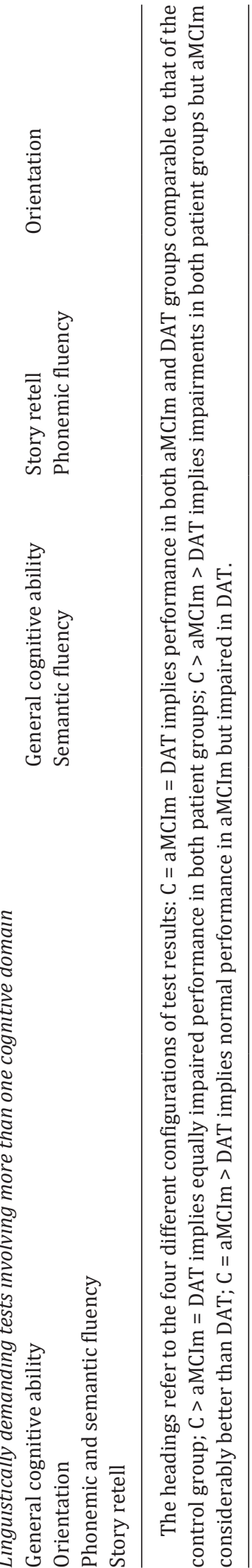




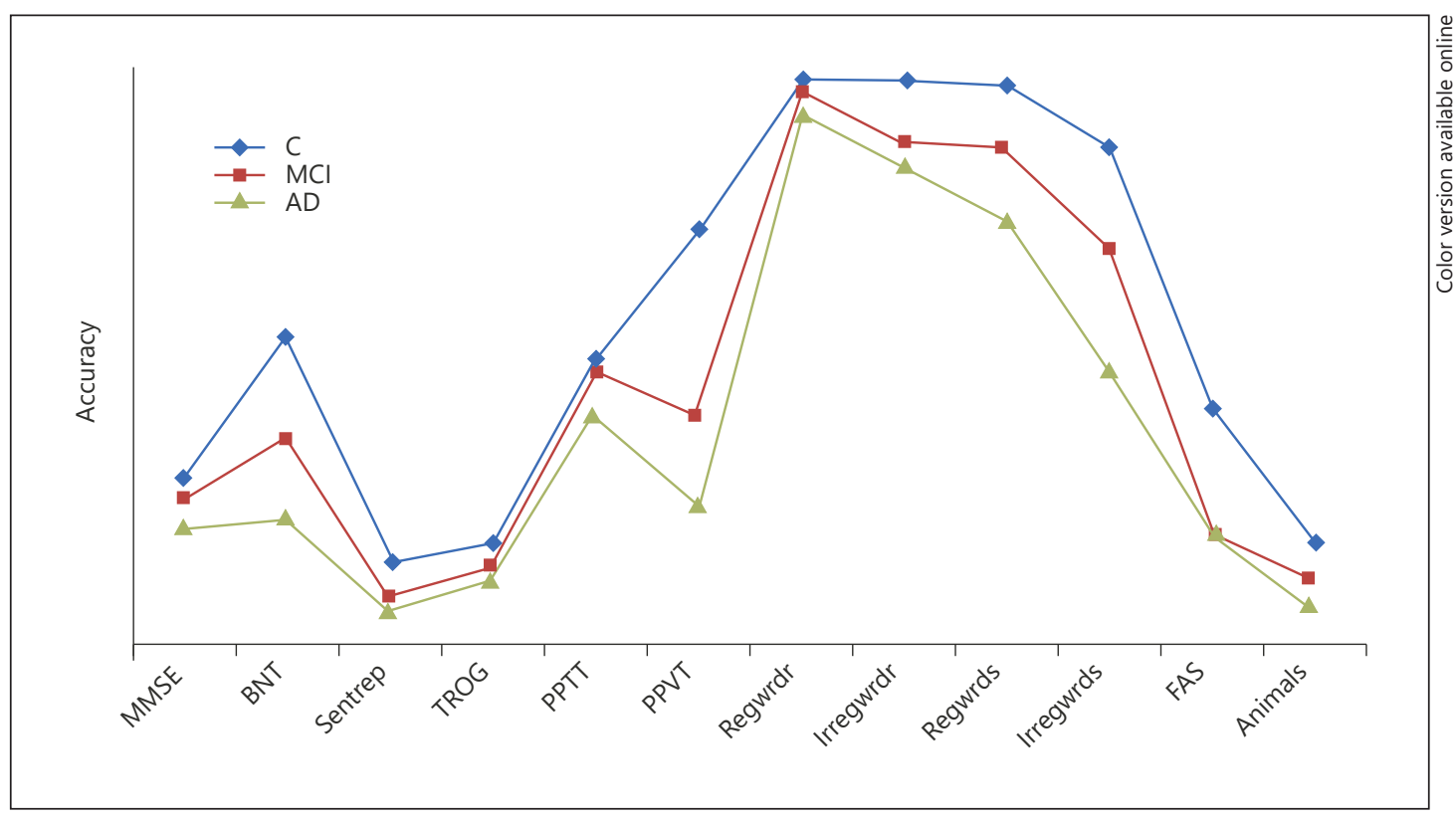

Fig. 1. Results of language tests in all groups. C, controls; MCI, mild cognitive impairment; AD, Alzheimer's disease; MMSE, Mini Mental State Examination; BNT, Boston Naming Test; Sentrep, sentence repetition; TROG, Test for the Reception of Grammar; PPTT, Pyramids and Palm Trees Test; PPVT, Peabody Picture Vocabulary Test; Reg, regular; Irreg, irregular; wrd, words; r, reading; s, spelling; FAS, phonemic fluency test with letters f, a and s; animals, semantic fluency tests.

Visual examination of profiles (Fig. 1) suggests that there is natural progression from normal ageing through aMCIm to DAT with naming and receptive vocabulary being most vulnerable to the decline.

\section{Discussion}

Language profiles of two groups of individuals with cognitive impairments due to aMCIm and DAT, respectively, were evaluated and compared to control data. The goal was to determine areas of language impairment in the two patient groups and establish whether potential differences would be qualitative or quantitative in nature, leading to the extraction of clinically helpful information. Predominantly quantitative differences emerged, consistent with parallel profiles of language impairment across the board, different only in the level of impairment. Although in no case results in the DAT group were superior to those in the MCI group, there were four different configurations of test results: (i) normal performance $(\mathrm{N})$ in both aMCIm and DAT group, $\mathrm{N}=$ aMCIm = DAT (word reading irrespective of spelling regularity, oral passage reading and spelling of regular words), (ii) equally impaired performance in both groups, N > aMCIm = DAT (story retell, receptive grammar and phonemic fluency), (iii) both groups impaired but aMCIm considerably less than DAT, N > aMCIm > DAT (MMSE, naming, spelling of irregular words, semantic fluency and, marginally, sentence repetition), (iv) normal performance in aMCIm but impaired in DAT, $\mathrm{N}=$ aMCIm $>$ DAT (orientation, verbal and non-verbal semantic access).

General mental function, as measured with the MMSE, was impaired in the DAT group and better preserved in the aMCIm group. These profiles have been well documented for both 
populations and are consistent with the amount of brain atrophy and functional independence in each group as reported in the literature $[63,64]$. Of note, the MMSE has been proven to be less sensitive in identifying cognitive impairments in MCI than other cognitive measures, such as the Montreal Cognitive Assessment [65]. Oral story recall, reliant on episodic and working memory, was impaired in both groups. Story recall is the only task included in this study that equally taxes language and memory functioning and one that can also reliably identify impairments in memory. Working memory impairments were evident on the sentence repetition task in both patient groups.

Some sources argue that, although semantic memory is generally preserved in $\mathrm{MCI}$, lexical production is impaired on tasks requiring effortful semantic memory processing [66], such as fluency and naming tasks. Both patient groups in our study were impaired on naming and semantic fluency compared to normative data but the aMCIm group showed less severe impairments at a comparable point after the onset. Of note, phonemic fluency and story retell/recall have some processes in common with receptive language - in particular sequencing and strategic search. Naming and semantic fluency have recently been identified in the literature as having high predictive value in conversion from MCI to DAT [for a review, see 67]. Naming may, in fact, have a better predictive value than memory, even though naming may initially appear to be intact in MCI. Furthermore, the notion that word finding difficulties are present often and early in DAT $[22,23]$ was confirmed by our results. In addition, consistent with the literature $[25,26]$, both aMCIm and DAT groups showed a severer decline in semantic fluency when compared to phonemic fluency. Yet access to semantic knowledge from both verbal and non-verbal cues was largely intact in our aMCIm group. It appears that accessing the phonology may be troublesome in aMCIm, which appears consistent with the tip-of-thetongue phenomenon characteristic of healthy aging and the transition period between MCI and DAT. Participants with DAT present with impaired access to phonology and additional semantic deficits. This suggestion is consistent with the DAT group's impaired performance on a non-verbal semantic test, the Pyramids and Palm Trees Test. While both controls and participants with aMCIm performed within the normal range, individuals with DAT showed clear impairment while trying to negotiate the most salient features of items to be matched.

Oral reading of regular words and a passage, although slower than in the control group, was relatively normal in both patient groups. These tasks are likely less affected by cognitive decline since they do not require episodic and semantic memory. Some semantic memory may be required to correctly read irregular words; however, successful mapping between the orthographic input lexicon and phonological output lexicon [58] can also be accomplished in a more automatic manner. Cognitive demands on a reading task that does not require comprehension can be fulfilled relatively easily, even in a mildly compromised system. In contrast, written spelling of irregular words requires cognitive resources, such as accessing the intact orthographic output buffer which is equated in cognitive neuropsychology with working memory [58]. Accordingly, impairments were found in both aMCIm and DAT groups. Thus, spelling of irregular words to dictation can be used as a relatively sensitive language tool in the assessment of cognitive decline in dementia.

Another task that emerged as a potential marker of cognitive decline was the comprehension of syntax (TROG) which was impaired in both aMCIm and DAT groups. This is in disagreement with a study by Lambon Ralph et al. [52] in which MCI participants performed as well as control participants. Successful understanding of various syntactic relations occurs at several levels and necessitates recruitment of a number of cognitive processes. In addition to preserved syntactic knowledge, it also requires intact working memory, especially for complex structures, such as centre-embedded sentences [68]. Working memory deficits in DAT were already documented decades ago [69], so, with the addition of more recent evidence of deficits in receptive syntax $[50,51]$, it comes as no surprise that individuals with DAT show 
impairments on tests of syntactic comprehension on the TROG. In-depth studies of syntactic function, either receptive or expressive, in the MCI population have not been conducted to allow us to make conclusions about which aspects of memory/language function may be responsible for our findings. We can speculate that subtle deficits in working memory and, possibly, attentional-executive processing may be responsible, given that both groups made errors predominantly on the centre-embedded relative clause.

Overall, this study provided evidence for relative preservation of aspects of expressive language in both aMCIm and DAT, although the measures used here did not address all aspects of verbal expression and future studies may identify other sensitive tasks and further deficits. Early studies of discourse and narrative in DAT have already informed us on possible impairments in cohesion and coherence. In contrast to DAT, orientation to time and place remains preserved in aMCIm as are many aspects of semantic knowledge. In addition, in both aMCIm and DAT, impairments are apparent in receptive language and in linguistically complex tests requiring other cognitive domains, such as story retell (needs episodic memory) and verbal fluency (needs executive functions). Both tasks may additionally share sequencing and strategic search demands inherent in receptive language tasks.

\section{Study Limitations}

Sample size is an omnipresent issue in studies of language in disordered populations, and our study is not immune to that. In addition, although each participant in the MCI and AD group received consensus diagnosis for their respective disorder, the lack of perfect homogeneity among participants, evident on test results, may present another limitation.

\section{Conclusion}

The purpose of this study was to explore language differences between individuals diagnosed with aMCIm and those with probable AD, with a goal of (i) characterizing the language profile of aMCIm and (ii) determining whether the profiles of DAT and aMCIm individuals are on a continuum of one diagnostic entity or represent two distinct cognitive disorders. The results are suggestive of a continuum between aMCIm and typical DAT whereby some functions may decline earlier and faster than others. The study supports utilizing language assessment (story retell, syntax comprehension and spelling of irregular words, in particular) as an integral part of cognitive evaluation in a diagnostic work-up of individuals presenting with an insidious onset of cognitive decline. Studies involving other $\mathrm{MCI}$ subtypes with exploration of factors that may be associated with faster conversion rate and earlier identification may further assist with managing cognitive disorders of ageing. Certainly, understanding patterns of language decline would be expected to critically inform cognitive intervention research with older adults living with MCI and DAT. Characterization of expressive and receptive deficits would help in shaping management strategies and utilizing preserved aspects of verbal and non-verbal communication.

\section{Statement of Ethics}

All healthy control participants provided informed consent. Ethics approval was granted for retrospective chart review. The study protocol has been approved by the Baycrest Research Ethics Board. 
Jokel et al.: Language in $\mathrm{MCl}$ and DAT

\section{Disclosure Statement}

The authors have no conflicts of interest to declare.

\section{Funding Sources}

Regina Jokel and Alita Fernandez were supported by the Alzheimer's Association/Canada Brain and CABHI. Bruna Seixas Lima was support by a Canadian Institutes of Health Research doctoral fellowship, and Kelly J. Murphy was supported by the Morris Goldenberg Medical Research Endowment.

\section{Author Contributions}

Regina Jokel initiated the study, conducted chart reviews, supervised data collection and analysis, and prepared the initial and revised versions of the manuscript, including figures and tables. Bruna Seixas Lima carried out the initial literature review. Alita Fernandez collected data from healthy controls and analysed the data from all participants. Kelly Murphy contributed her expertise in $\mathrm{MCI}$ to the introduction and discussion sections. All authors participated in editing the final version of the manuscript.

\section{References}

1 Cummings JL. Alzheimer’s disease. N Engl J Med. 2004 Jul;351(1):56-67.

2 Koch G, Bonnì S, Pellicciari MC, Casula EP, Mancini M, Esposito R, et al. Transcranial magnetic stimulation of the precuneus enhances memory and neural activity in prodromal Alzheimer's disease. Neuroimage. 2018 Apr;169:302-11.

3 Becker JT, Overman AA. The memory deficit in Alzheimer's disease. In: Baddeley AD, Kopelman MD, Wilson BA, editors. The handbook of memory disorders. 2nd ed. Chichester: Wiley \& Sons; 2002. p. 569-589.

4 Petersen RC, Caracciolo B, Brayne C, Gauthier S, Jelic V, Fratiglioni L. Mild cognitive impairment: a concept in evolution. J Intern Med. 2014 Mar;275(3):214-28.

5 Albert MS, DeKosky ST, Dickson D, Dubois B, Feldman HH, Fox NC, et al. The diagnosis of mild cognitive impairment due to Alzheimer's disease: recommendations from the National Institute on Aging-Alzheimer's Association workgroups on diagnostic guidelines for Alzheimer's disease. Alzheimers Dement. 2011 May; $7(3): 270-9$.

6 Flicker C, Ferris SH, Reisberg B. Mild cognitive impairment in the elderly: predictors of dementia. Neurology. 1991 Jul;41(7):1006-9.

7 Petersen RC, Smith GE, Waring SC, Ivnik RJ, Tangalos EG, Kokmen E. Mild cognitive impairment: clinical characterization and outcome. Arch Neurol. 1999 Mar;56(3):303-8.

8 Knopman DS, Boeve BF, Petersen RC. Essentials of the proper diagnoses of mild cognitive impairment, dementia, and major subtypes of dementia. Mayo Clin Proc. 2003 Oct;78(10):1290-308.

9 Anderson ND, Murphy KJ, Troyer AK. Living with mild cognitive impairment. Oxford: Oxford University Press; 2012.

10 Bruscoli M, Lovestone S. Is MCI really just early dementia? A systematic review of conversion studies. Int Psychogeriatr. 2004 Jun;16(2):129-40.

11 DeCarli C, Mungas D, Harvey D, Reed B, Weiner M, Chui H, et al. Memory impairment, but not cerebrovascular disease, predicts progression of MCI to dementia. Neurology. 2004 Jul;63(2):220-7.

12 Maioli F, Coveri M, Pagni P, Chiandetti C, Marchetti C, Ciarrocchi R, Ruggero C, Nativio V, Onesti A, D'Anastasio C, Pedone V. Conversion of mild cognitive impairment to dementia in elderly subjects: a preliminary study in a memory and cognitive disorder unit. Arch Gerontol Geriatr. 2007;44 Suppl 1:233-41.

13 Gorno-Tempini ML, Brambati SM, Ginex V, Ogar J, Dronkers NF, Marcone A, et al. The logopenic/phonological variant of primary progressive aphasia. Neurology. 2008 0ct; 71(16):1227-34.

14 Henry ML, Gorno-Tempini ML. The logopenic variant of primary progressive aphasia. Curr Opin Neurol. 2010 Dec;23(6):633-7. 
Jokel et al.: Language in $\mathrm{MCl}$ and DAT

15 Ahmed S, de Jager CA, Haigh AM, Garrard P. Logopenic aphasia in Alzheimer's disease: clinical variant or clinical feature? J Neurol Neurosurg Psychiatry. 2012 Nov;83(11):1056-62.

16 Gorno-Tempini ML, Hillis AE, Weintraub S, Kertesz A, Mendez M, Cappa SF, et al. Classification of primary progressive aphasia and its variants. Neurology. 2011 Mar;76(11):1006-14.

17 Teichmann M, Kas A, Boutet C, Ferrieux S, Nogues M, Samri D, et al. Deciphering logopenic primary progressive aphasia: a clinical, imaging and biomarker investigation. Brain. 2013 Nov;136(Pt 11):3474-88.

18 Ahmed S, Haigh AM, de Jager CA, Garrard P. Connected speech as a marker of disease progression in autopsyproven Alzheimer's disease. Brain. 2013 Dec;136(Pt 12):3727-37.

19 Verdelho A, Hénon H, Lebert F, Pasquier F, Leys D. Depressive symptoms after stroke and relationship with dementia. A three-year follow-up study. Neurology. 2004 Mar 23;62(6):905-11.

20 Starkstein SE, Robinson RG. Aphasia and depression. Aphasiology. 1988;2:1-20.21.

21 De Vriendt P, Gorus E, Cornelis E, Velghe A, Petrovic M, Mets T. The process of decline in advanced activities of daily living: a qualitative explorative study in mild cognitive impairment. Int Psychogeriatr. 2012 Jun;24(6): 974-86.

22 Appell J, Kertesz A, Fisman M. A study of language functioning in Alzheimer patients. Brain Lang. 1982 Sep; 17(1):73-91.

23 Bayles KA, Tomoeda CK. Confrontation naming impairment in dementia. Brain Lang. 1983;19(1):98-114.

24 Duong A, Whitehead V, Hanratty K, Chertkow H. The nature of lexico-semantic processing deficits in mild cognitive impairment. Neuropsychologia. 2006;44(10):1928-35.

25 Henry JD, Crawford JR, Phillips LH. Verbal fluency performance in dementia of the Alzheimer's type: a metaanalysis. Neuropsychologia. 2004;42(9):1212-22.

26 Murphy KJ, Rich JB, Troyer AK. Verbal fluency patterns in amnestic mild cognitive impairment are characteristic of Alzheimer's type dementia. J Int Neuropsychol Soc. 2006 Jul;12(4):570-4.

27 Östberg P, Fernaeus SE, Hellström K, Bogdanović N, Wahlund LO. Impaired verb fluency: a sign of mild cognitive impairment. Brain Lang. 2005 Nov;95(2):273-9.

28 Tierney MC, Yao C, Kiss A, McDowell I. Neuropsychological tests accurately predict incident Alzheimer disease after 5 and 10 years. Neurology. 2005 Jun;64(11):1853-9.

29 Molinuevo JL, Gómez-Anson B, Monte GC, Bosch B, Sánchez-Valle R, Rami L. Neuropsychological profile of prodromal Alzheimer's disease (Prd-AD) and their radiological correlates. Arch Gerontol Geriatr. 2011 Mar-Apr;52(2):190-6.

30 Venneri A, Gorgoglione G, Toraci C, Nocetti L, Panzetti P, Nichelli P. Combining neuropsychological and structural neuroimaging indicators of conversion to Alzheimer's disease in amnestic mild cognitive impairment. Curr Alzheimer Res. 2011 Nov;8(7):789-97.

31 Clark DG, Kapur P, Geldmacher DS, Brockington JC, Harrell L, DeRamus TP, et al. Latent information in fluency lists predicts functional decline in persons at risk for Alzheimer disease. Cortex. 2014 Jun;55:202-18.

32 Clark DG, McLaughlin PM, Woo E, Hwang K, Hurtz S, Ramirez L, et al. Novel verbal fluency scores and structural brain imaging for prediction of cognitive outcome in mild cognitive impairment. Alzheimers Dement. 2016;2: 113-22.

33 Borg C, Bedoin N, Basaglia-Pappas S, Laroche L, Minvielle B, Chesnoy G, et al. Semantic knowledge of newly coined words in mild cognitive impairment and Alzheimer's disease. J Neurolinguist. 2012;25(4):263-75.

34 Constantinidou F, Zaganas I, Papastefanakis E, Kasselimis D, Nidos A, Simos PG. Age-related decline in verbal learning is moderated by demographic factors, working memory capacity, and presence of amnestic mild cognitive impairment. J Int Neuropsychol Soc. 2014 Sep;20(8):822-35.

35 Cardoso S, Silva D, Maroco J, de Mendonça A, Guerreiro M. Non-literal language deficits in mild cognitive impairment. Psychogeriatrics. 2014 Dec;14(4):222-8.

36 Leyhe T, Saur R, Eschweiler GW, Milian M. Impairment in proverb interpretation as an executive function deficit in patients with amnestic mild cognitive impairment and early Alzheimer's disease. Dement Geriatr Cogn Disord Extra. 2011 Jan;1(1):51-61.

37 Yamaguchi H, Maki Y, Yamaguchi T. A figurative proverb test for dementia: rapid detection of disinhibition, excuse and confabulation, causing discommunication. Psychogeriatrics. 2011 Dec;11(4):205-11.

38 Gaudreau G, Monetta L, Macoir J, Laforce R Jr, Poulin S, Hudon C. Verbal irony comprehension in older adults with amnestic mild cognitive impairment. Neuropsychology. 2013 Nov;27(6):702-12.

39 Murphy KJ, Troyer AK, Levine B, Moscovitch M. Episodic, but not semantic, autobiographical memory is reduced in amnestic mild cognitive impairment. Neuropsychologia. 2008 Nov;46(13):3116-23.

40 Irish M, Kamminga J, Addis DR, Crain S, Thornton R, Hodges JR, et al. 'Language of the past' - Exploring past tense disruption during autobiographical narration in neurodegenerative disorders. J Neuropsychol. 2016 Sep;10(2):295-316.

41 Dijkstra K, Bourgeois MS, Allen RS, Burgio LD. Conversational coherence: discourse analysis of older adults with and without dementia. J Neurolinguist. 2004;17(4):263-83.

42 Harris JL, Kiran S, Marquardt TP, Fleming VB. Communication Wellness Check-Ups: age-related changes in communicative abilities. Aphasiology. 2008;22(7-8):813-25.

43 Fleming VB, Harris JL. Complex discourse production in mild cognitive impairment: detecting subtle changes. Aphasiology. 2008;22(7-8):729-40. 
Drummond C, Coutinho G, Fonseca RP, Assunção N, Teldeschi A, de Oliveira-Souza R, et al. Deficits in narrative discourse elicited by visual stimuli are already present in patients with mild cognitive impairment. Front Aging Neurosci. 2015 May; 7:96.

45 Radanovic M, Mansur LL. Performance of a Brazilian population sample in the Boston Diagnostic Aphasia Examination: a pilot study. Braz J Med Biol Res. 2002 Mar;35(3):305-17.

46 Taler V, Phillips NA. Language performance in Alzheimer's disease and mild cognitive impairment: a comparative review. J Clin Exp Neuropsychol. 2008 Jul;30(5):501-56.

47 Bayles KA, Kaszniak AW, Tomoeda CK. Communication and cognition in normal aging and dementia. Boston: College-Hill Press/Little, Brown \& Co.; 1987.

48 Fisher NJ, Tierney MC, Rourke BP, Szalai JP. Verbal fluency patterns in two subgroups of patients with Alzheimer's disease. Clin Neuropsychol. 2004 Feb;18(1):122-31.

49 Kemper S, LaBarge E, Ferraro FR, Cheung H, Cheung H, Storandt M. On the preservation of syntax in Alzheimer's disease. Evidence from written sentences. Arch Neurol. 1993 Jan;50(1):81-6.

50 Fyndanis V, Manouilidou C, Koufou E, Karampekios S, Tsapakis EM. Agrammatic patterns in Alzheimer's disease: evidence from tense, agreement and aspect. Aphasiology. 2013;27(2):178-200.

51 Auclair-Ouellet N. Inflectional morphology in primary progressive aphasia and Alzheimer's disease: a systematic review. J Neurol. 2015;34:41-64.

52 Lambon Ralph MA, Patterson K, Graham N, Dawson K, Hodges JR. Homogeneity and heterogeneity in mild cognitive impairment and Alzheimer's disease: a cross-sectional and longitudinal study of 55 cases. Brain. 2003 Nov; 126(Pt 11):2350-62.

53 McKhann GM, Knopman DS, Chertkow H, Hyman BT, Jack CR Jr, Kawas CH, et al. The diagnosis of dementia due to Alzheimer's disease: recommendations from the National Institute on Aging-Alzheimer's Association workgroups on diagnostic guidelines for Alzheimer's disease. Alzheimers Dement. 2011 May;7(3):263-9.

54 Folstein MF, Folstein SE, McHugh PR. "Mini-mental state". A practical method for grading the cognitive state of patients for the clinician. J Psychiatr Res. 1975 Nov;12(3):189-98.

55 Kaplan E, Goodglass H, Weintraub S. The Boston Naming Test. Boston. Philadelphia (PA): Lippincott, Williams \& Wilkins; 2001.

56 Goodglass H, Kaplan E, Barresi E. Boston Diagnostic Aphasia Examination. Philadelphia (PA): Lippincott, Williams \& Wilkins; 2001.

57 Bayles KA, Tomoeda CK. Arizona Battery for Communication Disorders of Dementia. Austin (TX): PRO-ED; 1993.

58 Kay J, Lesser R. Coltheart M. Psycholinguistic Assessment of Language Processing in Aphasia. Hove, UK: Lawrence Erlbaum Associates Ltd.; 1992.

59 Bishop DV. Test for the Reception of Grammar (TROG-2)-Version 2. London: Psychological Corporation; 2003.

60 Dunn M, Dunn LM. Peabody Picture Vocabulary Test-4. Circle Pines (MN): AGS; 2007.

61 Howard D, Patterson K. The Pyramids and Palm Trees Test. Burry St Edmunds, UK: Thames Valley Test Company; 1992.

62 Tombaugh TN, Kozak J, Rees L. Normative data stratified by age and education for two measures of verbal fluency: FAS and animal naming. Arch Clin Neuropsychol. 1999 Feb;14(2):167-77.

63 Leung KK, Bartlett JW, Barnes J, Manning EN, Ourselin S, Fox NC; Alzheimer's Disease Neuroimaging Initiative. Cerebral atrophy in mild cognitive impairment and Alzheimer disease: rates and acceleration. Neurology. 2013 Feb;80(7):648-54.

64 Jack CR Jr, Shiung MM, Gunter JL, O’Brien PC, Weigand SD, Knopman DS, et al. Comparison of different MRI brain atrophy rate measures with clinical disease progression in AD. Neurology. 2004 Feb;62(4):591-600.

65 Trzepacz PT, Hochstetler H, Wang S, Walker B, Saykin AJ; Alzheimer's Disease Neuroimaging Initiative. Relationship between the Montreal Cognitive Assessment and Mini-mental State Examination for assessment of mild cognitive impairment in older adults. BMC Geriatr. 2015 Sep;15(1):107.

66 Duong A, Whitehead V, Hanratty K, Chertkow H. The nature of lexico-semantic processing deficits in mild cognitive impairment. Neuropsychologia. 2006;44(10):1928-35.

67 Belleville S, Fouquet C, Hudon C, Zomahoun HT, Croteau J. Neuropsychological measures that predict progression from mild cognitive impairment to Alzheimer's type dementia in older adults: a systematic review and meta-analysis. Neuropsychol Rev. 2017;27(4):328-53.

68 Caplan D, Waters GS. Verbal working memory and sentence comprehension. Behav Brain Sci. 1999 Feb;22(1): 77-94.

69 Baddeley A, Logie R, Bressi S, Della Sala S, Spinnler H. Dementia and working memory. Q J Exp Psychol A. 1986 Nov;38(4):603-18. 